\title{
Child neurology in the 21st century
}

\section{More than the sum of our RVUs}

Mary L. Zupanc, MD, Bruce H. Cohen, MD, Peter B. Kang, MD, David E. Mandelbaum, MD, PhD,
Jonathan Mink, MD, Mark Mintz, MD, Ann Tilton, MD, and William Trescher, MD

Neurology ${ }^{\circledR}$ 2020;94:75-82. doi:10.1212/WNL.0000000000008784

\section{Abstract}

In September 2017, the Child Neurology Society (CNS) convened a special task force to review the practice of child neurology in the United States. This was deemed a necessity by our membership, as our colleagues expressed discouragement and burnout by the increase in workload without additional resources; reliance on work relative value units (wRVUs) as the sole basis of compensation; a push by administrators for providers to see more patients with less allotted time; and lack of administrative, educational, and research support. The CNS Task Force designed and distributed a survey to multiple academic divisions of various sizes, as well as to private practices. Our findings were strikingly similar across different practices, demonstrating high workloads, lack of resources, poor electronic medical record support, and high provider symptoms of fatigue and burnout. From the results, the CNS Task Force has concluded that wRVUs cannot be the sole basis of compensation for child neurology. We have also made several specific recommendations for alleviating the current situation, including innovative ways to fund child neurology as well as ways to enhance job satisfaction.

\section{Introduction}

Child neurologists evaluate children with complex medical issues, whose care cannot be managed in a typical 15- to 20-minute pediatric visit. In addition, they must counsel parents who are under stress and have a multitude of questions. These patients typically require multidisciplinary team care and ongoing case management to attain optimum outcomes and quality of life. The time required for this intensive, diagnostic approach and multidisciplinary care plan not only results in better direct outcomes for the child and family, but also in decreased emergency room visits, fewer unscheduled hospitalizations, and less burden on the primary care provider.

A developing critical shortage of child neurologists is making patient access difficult and increasing the burden on the remaining and strained workforce. Increasing the number of child neurologists is therefore essential for the future of children with neurologic disorders. To help increase this number, child neurologists spend many hours training the future generation of child neurologists and other health care providers. However, this educational mission is only possible with the proper support structure and with the recognition that these efforts will affect clinical productivity.

All physicians have a practice ideal of how to manage their patients clinically, viewing care as a mission. Failing to consistently meet patient needs has a profound effect. As stated in one article, ${ }^{1}$ physicians are not burning out, they are experiencing "moral injury"-where there is direct conflict between the increasingly business-oriented and profit-driven health care environment and the physician's desire to provide optimal care. On a personal level, this conflict can mean physical and emotional exhaustion, resulting in difficult choices such as restricting

\section{Correspondence}

Dr. Zupanc

mzupanc@choc.org

\section{RELATED ARTICLE}

\section{Editorial}

Putting value back into the "V" of wRVU

Page 57 


\section{Glossary}

CNS = Child Neurology Society; FTE = full-time employees; IPOP = independent private organizations and practices; NP = nurse practitioners; $\mathbf{R V U}=$ relative value unit; $\mathbf{w R V U}$ = work relative value unit.

one's practice, curtailing all commitments except direct patient care, leaving the academic setting, setting up a cashonly practice, or early retirement. On a societal level, it can result in the unwillingness of physicians-in-training to take on the burdens involved in this subspecialty and the premature loss of practicing child neurologists.

At most medical centers, a well-defined, uniform patientschedule template for all neurologists, regardless of subspecialty, removes variability and for many practices, establishes an agreement between the physician and employer about how much clinical work a physician will be expected to perform. The work relative value unit (wRVU), as a system of work value, has been developed and verified as a measure of work. Although the fairness of work value between a cognitive service such as an office visit and a procedure such as an appendectomy can be disputed, the work, as estimated by the annual wRVUs when comparing neurologists who perform only evaluation/management services, should be valid.

In September 2017, the Child Neurology Society (CNS) convened a special task force to review the practice of child neurology in the United States. The CNS relative value unit (RVU) Task Force designed and distributed a survey that queried child neurologists from both academic and private practices. The survey covered the use of RVU requirements, salary and compensation, time allotted for new and established patients, administrative and teaching mandates, clinical and administrative support, weekly face-to-face patient time, protected time, and educational opportunities. The aim was to define the challenges faced by child neurologists in providing optimum patient care and fulfilling the academic mission of research and education in today's health care marketplace. We surveyed 25 academic child neurology programs of diverse sizes as well as independent private organizations and practices (IPOP). In our initial analysis of the data, we identified common themes in each division or practice surveyed. We summarize our data below and offer specific recommendations.

\section{CNS RVU task force data summary gathered specifically from child neurology divisions}

Of the 25 academic programs surveyed, 20 provided enough detail for a complete data analysis.

The programs were divided into small, medium, and large programs. The small programs ${ }^{2}$ were composed of less than 5 faculty. The medium programs (12) contained 7-18 faculty with the majority of programs aggregating between 12 and 14 (median 13). The large programs ${ }^{6}$ were composed of over 20 faculty, varying between 22 and 64 .

There were no substantial geographic differences between salaries among the various programs, with the exception being a smaller salary scale (junior vs senior) in the larger programs.

1. Salaries for 1.0 full-time employees (FTE) ranged from $\$ 175,000$ to $\$ 350,000$ (table e-1, http://links.lww.com/ $\mathrm{WNL} / \mathrm{B} 41)$.

- Small programs:

Median and mean salary of $\$ 210,000$

- Medium programs:

Junior faculty—median salary $\$ 191,500$; mean salary \$191, 917

Senior faculty—median salary $\$ 273,500$; mean salary $\$ 285,455$

- Large programs:

Junior faculty—-median salary $\$ 165,000$; mean salary $\$ 164,400$

Senior faculty - median salary $\$ 290,000$; mean salary $\$ 271,000$

2. In $100 \%$ of programs surveyed, salary increases were based on years of experience or academic rank, but bonus structures varied considerably, as described in no. 4.

3. Minimum wRVU requirements for 1.0 FTE positions were variable(table e-2, http://links.lww.com/WNL/B41):

- Small programs:

Mixed: 4,891 wRVUs (range 4,800-4,981)

- Medium programs:

General neurology: 3,060 wRVUs (range 2,200-3,800)

Mixed: 4,365 wRVUs (range 4,000-4,980)

Epilepsy/proceduralist: 4,490 wRVUs (range $3,200-6,000$ )

- Large programs:

General neurology: 3,309 wRVUs (range 3,023-3,500)

Mixed: 4,200 wRVUs (1 program only)

Epilepsy/proceduralist: 4,361 wRVUs (range $3,900-7,177)$

4. A financial bonus was available in most divisions and was generally rewarded for exceeding wRVU targets. Clinical neurophysiologists (procedure-based pediatric neurologists) could have bonuses exceeding $\$ 150,000$.

- Most bonuses were generated by exceeding wRVU targets:

- One program used a pooled bonus divided equally among the faculty. 
- Many programs had a fixed base with incentive, incentive primarily wRVU based, with a small amount for educational effort and teaching, as well as research.

- Minor incentives were given for patient satisfaction and citizenship (e.g., membership on hospital or university committees).

- Some programs had bonuses fixed to wRVU target. If the physician exceeded the wRVU target, the physician would receive between $\$ 30$ and $\$ 40$ / wRVU above the target.

- In one large program, there was a tiered approach to distribution of bonuses (clinical participation $=60 \%$; service in pursuit of academic, clinical, educational mission $=20 \%$; discretionary $=20 \%$ ).

- One medium program provided bonuses determined by $50 \%$ productivity and $50 \%$ service.

- One program had no bonuses, with $85 \%$ fixed salary and $15 \%$ at risk, depending on whether the physician met the targeted wRVU goal.

- Two medium academic programs, or $10 \%$ of all programs surveyed, had no bonus structure.

- Overall, in those 18 programs with bonus structure:

- A total of $9 / 18$ or $50 \%$ of programs had a bonus structure based solely on wRVUs.

- A total of $9 / 18$ or $50 \%$ of programs had a bonus structure based on wRVUs, with small incentives for teaching, research, citizenship, and patient satisfaction.

- One program divided the pooled bonus equally among faculty with small incentives for citizenship, education, research, and significantly high volume of wRVUs.

5. One institution surveyed had a reduction in salary. The faculty of this program were required to pay back the university at the end of the fiscal year if wRVU targets were not met.

6. Clinic visits: Most programs allowed 60 minutes for new patients and 30 minutes for established patients.

- Small programs: $100 \%$ had 60/30 minutes.

- Medium programs: $10 / 12$ or $84 \%$ of programs had $60 /$ 30 minutes.

- Large programs: $5 / 6$ or $84 \%$ of programs had $60 / 30$ minutes.

7. Clinic sessions: Most programs required 7-8 sessions per week (half day), with 32 hours of actual face time/week. Many hospital/clinic administrators are pushing for 36 hours of actual face time/week(table e-3, http://links.lww. com/WNL/B41).

- Small programs had 6 sessions per week.

- Medium programs had an average of 7.75 sessions per week.

- Large programs (1 FTE) required 7 sessions per week.

8. Most programs received no educational support. Only one program was given $10 \% \mathrm{wRVU}$ credit for education. Ninety percent of programs had minimal or no educational support, except for variable support for the residency program director, as mandated by the Accreditation Council for Graduate Medical Education (Residency Review Committee requirements). One program provided $5 \%$ administrative support, $5 \%$ teaching support, and $10 \%$ initial research support for the faculty.

9. One program, out of the 25 surveyed, provided $10 \%$ initial research support. The remaining programs required faculty to obtain grants/outside research funding for research support.

10. Challenges:

- Increasing clinical demands with no additional resources

- Patient access, an issue for all divisions, with administrations often pushing for more face-to-face time

- The electronic medical record was identified as a stressor for physicians across all divisions and practices, especially with increasing documentation requirements mandated by Meaningful Use. Most physicians complete their notes at night or on weekends, with no additional wRVU credit. According to an American Academy of Neurology (AAN) survey, $31 \%$ of neurologists spend 1-4 hours per week after clinic completing their clinic notes; $34 \%$ of neurologists estimate they spend 5-9 hours per week after clinic completing their notes; $41 \%$ of neurologists estimate they spend over 10 hours per week after clinic completing their notes. ${ }^{2}$

- Variability of third party/insurance contracting

- Obstacles of regulations and authorizations for ordered services and medications

- There are large variances in physician support for patient care. For example, in some practices, a nurse performs almost all patient phone call activities, whereas in other practices, the physician is required to return all patient calls.

- Current Procedural Terminology has now created (and is creating more) new transition of care codes and non-faceto-face codes that can improve patient care. Physicians and hospital systems could use these codes to increase wRVU volume, but no practices surveyed have begun to utilize them, as they require changes in the standard operating procedure and infrastructure, including expensive information technology system builds that have not been implemented.

11. Provider burnout was reported as high among divisions, with all but one division reporting signs and symptoms of burnout in many of their faculty. In Neurology, Busis et al. ${ }^{6}$ discussed the 3 dimensions of burnout, including emotional exhaustion, depersonalization (feelings of cynicism and detachment), and a sense of ineffectiveness at work (low sense of personal accomplishment). The authors identified 6 domains associated with an increased risk of burnout, including workload, control, reward, community, fairness, and values. They also noted that burned out physicians may have impaired clinical judgement and lack of empathy, resulting in poor patient care and satisfaction. Furthermore, neurology is identified as one of the few specialties with both high rates of burnout and low satisfaction with work-life 
balance. Physician burnout among child neurologists is very similar to physician burnout in adult neurologists, estimated to be over $86 \%$. As a result, the physician workforce may shrink, due to attrition from clinical practice and fewer entering the specialty.

Our survey results are consistent with the findings identified by Busis et al. The burnout statistics from our survey are as follows: More than $90 \%$ of divisions identified some degree of burnout, as defined above, in their faculty. Perhaps more intriguing, $5 / 20$ or $25 \%$ of the division chiefs, while acknowledging burnout in their respective faculty, told us that "we don't discuss it."

In the 2 small programs, the physicians take call every night, read 2-6 EEGs per day, and have 6 sessions per week, with at least one symptom of burnout in every physician.

12. Nurse practitioners (NP), residents, and fellows augment the wRVU totals, artificially inflating the wRVUs for faculty who have less than a 1.0 FTE position (i.e., faculty with .3 FTE or .5 FTE positions who see patients with NP, residents, and fellows in addition to their own required clinic time).

13. Activities that do not generate revenue are as follows:

- Charting after the clinic visit

- Phone calls to patients or coordinating care after the day of service

- On-call responsibility that can be highly variable in work intensity and work frequency

- Teaching of medical students, residents, and fellows

- Peer-to-peer calls

- Medication changes and charting between office visits

- Reviewing laboratory and test results

- Reviewing records

- Hospital committee meetings and other mandatory meetings

- Coordinating care between visits (obtaining prior authorizations, reviewing laboratory and test results)

- Teaching and other mandatory conference attendance

- Continuing Medical Education activity

- Reviewing for academic journals

- Unfunded research and assisting trainees with their mandatory research

The results of the survey conducted by the CNS RVU task force reveal that, nationally, the expectation for a full-time, clinical faculty member is at 32 hours of patient face time per week. The time allotted for patients, while predominantly still at 60 minutes for a new patient and 30 minutes for follow-up, is under pressure to be reduced.

\section{Based on discussions of the CNS RVU}

\section{task force:}

1. The wRVU should not be used as the sole basis of compensation. Although the wRVU is a reasonable measure of work by which physicians performing the same type of work can be compared, it does not apply to physicians performing different types of work. The wRVU does not reflect the loss of sleep and additional stress required for taking night call and the work intensity of that night call. Child neurologists who are awakened repeatedly through the night and have no guaranteed sleep following their night call (which can extend for a week or more) do not generate any wRVUs during these nighttime hours, yet are performing a service that is critical to any hospital's function. In some hospital-based practices, there are no residents or fellows buffering the workload of the attending neurologist from this nighttime duty.

Furthermore, using the wRVU as a way to calculate the revenue generated by the physician's work is flawed. Both the technical wRVU and downstream revenues generated by that physician work, and the bed charges for those performing inpatient work, not only supports the total functioning of the hospital but also the physician's salary. For example, in a perfect but unrealistic scenario, a child neurologist with 32 hours face time/week spending 60 minutes for new patients and 30 minutes for established patients, working 46 weeks per year, billing at the highest consult and follow-up levels for all patients, with a $100 \%$ show rate, the wRVU generation would be 128 wRVUs per week $\times 46$ weeks for a total of 5,888 wRVUs per year. If each wRVU generated $\$ 54.17$ per wRVU (the calculation used at one of the free-standing children's hospitals) regardless of reimbursement, the highest possible revenue generated would be $\$ 318,952$. In addition, there will be another several hours of unreimbursed time related to the 32 hours face time/week and additional hours dedicated for on call duties. Medicaid contracts pay a fraction of that reimbursement. Therefore, the wRVU compensation model is both unrealistic and unsustainable, generating insufficient revenues to support reasonable compensation and not allowing time for non-patient-care duties. According to 2 colleagues on this committee, both of whom independently reviewed their faculty productivity and billing practices, the maximum wRVU generation per year for a non-procedurebased child neurologist was 3,200 wRVUs per year. Using the same calculation-that each wRVU would generate $\$ 54.17$ - the total revenue would be $\$ 173,344$, and half that value for practices with a predominant Medicaid population. This is not enough revenue to pay a physician salary when overhead ( $40 \%-50 \%$ of salary) and the cost of benefits ( $25 \%$ of salary) are considered.

Therefore, the contention that the wRVU should be the only measure of value to determine a physician's compensation is not valid, particularly if the mission of the practice, hospital, or medical center includes teaching, research, other scholarly activities, and community engagement. In harmony with present government trends of shifting from a fee-for-service to value-based models of reimbursement, models of measuring and determining physician value and compensation should evolve to alternative, innovative, and pragmatic prototypes that can eventually replace and eliminate the future use of wRVU.

2. The current wRVU expectations, using data obtained from Sullivan Cotter or ECG, based on 2018 survey data, place the 
wRVU expectations of child neurologists working at the 75th percentile at 4,827-4,935 wRVUs, the 90th percentile at 6,019-6,286 wRVUs, and the median percentile at 3,838-3,838 wRVUs. ${ }^{3,4}$ Most institutions mandate that child neurologists work at the 75th percentile or above. These $w R V U$ expectations, even at the median percentile, make it impossible to provide the quality of care expected of child neurologists, who are often asked to deal with complex problems that require time with the patient as well as the time to review the records and medical literature. This is especially true in small group practices that cannot afford having child neurologists with subspecialties in epilepsy, neuromuscular disorders, concussion, headache, and demyelinating disorders or in those child neurology practices that have general child neurologists who perform only a few procedures. Our CNS survey results differ from what is often presented by administrations as the mean RVU figure for child neurologists, raising valid questions about the basis for these figures.

3. Making wRVUs the sole basis of compensation is a design for failure with respect to promotions of junior or middle level faculty in academic centers, who are pursuing clinical research projects and fulfilling other requirements for academic advancement (see the table, criteria for promotion). By not providing alternative means of support, there will not be enough time to fulfill these criteria.

4. The ever-increasing problem of physician burnout is partly attributable to efforts to meet the demands for time spent with patients while also meeting the criteria for promotion. Physician burnout among child neurologists is very similar to physician burnout in adult neurologists, estimated to be over $86 \%{ }^{5,6}$ Our survey indicated that over $90 \%$ of child neurology academic divisions had faculty who had at least some signs or symptoms of burnout, as outlined in the data summary.

5. A substantial barrier to obtaining accurate data collection with respect to the average wRVU generation of child neurologists is statistical manipulation or misrepresentation when it comes to ECG and Sullivan-Cotter data. As an example, in a division with residents, fellows, or NP, and with faculty who have designated administrative time/ research time, the wRVU generation of a 1.0 FTE faculty becomes artificially inflated. Many of the queried child

Table Recommendations for criteria for promotion

\begin{tabular}{l}
\hline Criteria for promotion to clinical associate professor \\
\hline A high level of skill in teaching, advising, or mentoring, and as \\
a practitioner, and who has contributed actively to clinical or research \\
programs. The individual must have a regional reputation in his or her \\
area of expertise. Scholarly activity will be given positive consideration. \\
Service to the university, its affiliates, or to the profession will be given \\
positive consideration where a substantial role can be documented.
\end{tabular}

Criteria for promotion to clinical professor

Excellence in teaching, advising, or mentoring, and as a practitioner, and who has contributed actively to clinical or research programs. The individual must have a national reputation in his or her area of expertise. Evidence of scholarly activity is required. Service to the university, its affiliates, or to the profession will be given positive consideration where a substantial role can be documented. neurology divisions acknowledged this, noting, for example, a 0.3 FTE faculty member may add on additional clinics, staff a resident clinic, or sign off on NP clinic notes. This results in an artificially high wRVU for a part-time clinical faculty member, which, when extrapolated to a full 1.0 FTE, distorts the FTE RVU figure. (This may explain the discrepancy between our survey results and the figures for the mean RVU from other sources.)

6. In communities that may be considered less desirable by young child neurologists, typically those that are small or rural, the divisions can be composed of only 2-3 neurologists. Access is difficult, workloads are great, and pressure by administration high. These divisions often have difficulty retaining child neurologists. To recruit additional child neurologists and to avoid losing all their child neurologists, administrators often recruit child neurologists using high introductory salaries and reasonable work weeks (e.g., 6 half-day sessions or 24 hours of face time per week). Once these recruitment efforts are successful, there is increasing pressure to increase the work week, add on patients, and decrease the amount of time spent with new and return patients. Salaries are not progressive and stagnate. Often, the newly recruited child neurologists leave, resulting in a repetitive cycle. The administrators may understand this only after the damage is done and the child neurologists have left.

7. Salaries are regressing to the mean. There is not a progressive salary structure among child neurology divisions. There are larger initial salaries, but stagnation of salaries exists at the top, penalizing seniority.

8. The experience and wisdom of senior child neurologists cannot be easily translated into wRVUs-or, for that matter, time to next appointment or hospital readmission rates, for example.

9. We train the future generation of child neurologists, yet we receive no compensation for our educational activities.

10. Child neurology is a necessary service. A children's hospital cannot operate without child neurologists who perform consultations in the pediatric intensive care unit, neonatal intensive care unit, and medical-surgical floors or have residency programs that require child neurology training (to comply with regulatory mandates).

11. The cohort of child neurology patients is growing, while the workforce pool of child neurologists is diminishing.

\section{Recommendations}

For the sake of the children we care for, the students and residents we are charged to train, the members of the child neurology community, and the discipline of child neurology itself, we propose the following for academic settings:

1. The decisions regarding clinical productivity take place in a transparent discussion, considering the mission of the hospital, medical center, university, or other corporate 
structure, as well as the actual job description of the physician who will be delivering that care. Job descriptions based on human resource offices should be reviewed and updated to reflect the actual work and duties of each physician.

2. The consultant's statistical methods for determining average wRVU production should be disclosed. The current data appear to reflect a blend of private practice and academic practice, even in communities without private practice child neurology services, without any consideration of the difference between a neurologist who performs clinical neurophysiology vs a non-procedure-based, general neurologist.

3. wRVUs should not be used as the sole basis of compensation for child neurologists. There are other tangible and essential requirements that require time during the work day, and these should be accounted for in detail before coming to any firm decision regarding annual wRVU requirements. wRVUs can be used as part of the calculations, but other contributions should be included.

- Downstream revenue should be a part of the evaluation for compensation, using the mission statement and job descriptions. We were able to obtain downstream revenue data from 4 academic institutions. The collective downstream revenue per child neurologist (procedure-based combined with non-procedurebased) was estimated to be between $\$ 1.2$ and $\$ 2.0$ million dollars per child neurologist per year. These 4 institutions declined to be identified. In many institutions, these data are carefully guarded and difficult to obtain.

- Child neurology services that are essential to the maintenance of a functioning medical center do not generate wRVUs. Although many of these duties are not unique to this specialty, some of the duties are overrepresented in this specialty. This includes, but is not limited to, the volume of phone calls at night from the emergency department requesting phone consultation, phone calls at night from various intensive care units, and phone calls from colleagues during the day for curbside consultations, none of which generate wRVUs. Much of this work is performed at night, during normal sleep time, which is essential, but most programs have no method of tracking or reacting to sleep deprivation. Although there are health concerns and patient safety concerns that should be addressed separately, extra consideration should be given for reducing the wRVU requirement for those practices that require nighttime work more frequently. In addition, children entering our care will also ultimately be generating revenue for radiology, orthopedics, and other specialties within the organization. The downstream revenue argument works both ways but neurology, specifically, is the nidus of activity for entry into the medical system rather than a competitor's medical system.

- There needs to be recognition of the value of the input into the system that general child neurologists (nonproceduralists) bring. These general child neurologists feed the neuroscience enterprise. They are not a loss-leader scenario. Rather, these general child neurologists are an essential part of the corpus of child neurology. We also need to explain clearly to our respective institutions why the providers who serve and bring patients into the health care system cannot possibly meet the productivity measures set by ECG and Sullivan Cotter, given that they do not have procedures to increase their wRVUs. Furthermore, the productivity measures for general child neurologists do not consider the current deficiencies in scheduling, no-show rates, and same-day cancellations. None of these factors is under the control of the providers. Call responsibilities are also not accounted for adequately using the wRVU system, specifically, the need to be available $24 / 7$ and the many interruptions during the day and night while on call. Expectations for our general neurology providers must be realistic and achievable and recognize the deficiencies in our systems.

- If the mission of the employer/institution includes education or research, the clinical faculty without extramural research support should have reduced clinical time and wRVU requirements to enable them to achieve the criteria for promotion (table). Specifically, those full-time clinical faculty in an academic center, pursuing promotion without extramural research support, should be expected to do no more than 5 or 6 half-day clinical sessions per week (20-24 hours of face to face clinical time. The decision for clinical time should be determined by the child neurology division, not administration.

- Clinic schedules should allot 60 minutes for a new patient and 30 minutes for established patients.

- Clinic time should be reduced when the individual has inpatient care responsibilities.

- Metrics should be developed to provide adequate support services that allow child neurologists to practice at the top of their license, rather than performing duties that might best be completed by others.

- Clinical support staff are needed to respond to patient telephone calls, facilitate prescription refills, and complete forms (school forms, seizure action plans, Family Medical Leave Act forms, Supplemental Security Income forms).

- Scribes are needed to assist with completion of clinical notes at the time of the visit.

- Advanced practice providers (NP and physician assistants) are needed to to work collaboratively and independently with physicians to enhance access. Advanced practice providers are particularly capable in designing and managing subspecialty clinics, including headache clinics, first seizure clinics, and febrile seizure clinics.

- Administrative support is needed to relieve neurologists from administrative tasks such as prior 
authorization, faxing forms, taking information for prescription refills, and typing manuscripts.

- Social workers are needed to help families manage or gain access to insurance, financial support services, child assistance programs, and so forth.

- Educational psychologists are needed to assist families in obtaining appropriate educational services for their child.

- Providing education should be compensated. Some institutions have established academic/research and educational value units outside of clinic work to recognize these efforts. This practice should be widely implemented.

- Startup funds should be provided for clinical research, along with protected time.

- Global payment models, particularly if they are based on patient outcomes and the cost-effectiveness of multidisciplinary teams, should be utilized. There are several studies that clearly demonstrate the effectiveness of multidisciplinary teams, resulting in improvement in quality of life, better patient outcomes, decrease in emergency room visits and unscheduled hospital admissions, as well as medical cost savings. ${ }^{7,8}$

- Young junior clinical faculty are expected to make steady progress toward the criteria for promotion and should be given wRVU credit for this endeavor. If it is evident, after a mutually agreed time, that the faculty member is not pursuing activities required for promotion, an increase in clinical sessions to 7 or 8 four-hour clinical sessions per week ( 28 or 32 hours of direct clinical time) may be implemented. This can be reconsidered if the faculty member makes more progress toward promotion.

- Salary support derived from extramural sources will be used to decrease clinical time, facilitating research or academic activity.

\section{Independent/private organizations/practices}

In reviewing the surveys from multiple IPOP, there are many similarities to the academic pediatric neurology divisions, with a few caveats. As expected, most of the private practice neurologists have more robust scheduling, ranging from 8 to 10 half-day sessions per week, with each session ranging from 3.5 to 4 hours. As with many clinical child neurologists in academia, the physicians in private practice (IPOP) provide 45-60 hours per week in clinically related tasks, with $20 \%-30 \%$ not billed or reimbursed, performing the tasks outlined above. All surveyed IPOP are engaged to varying degrees in academic publications and presentations, clinical research, or education and training of medical students, residents, fellows, and others, making the distinction between academia and IPOP in this area hazy.

The primary differences between academic child neurologists and IPOP included the feeling of independence and control of their own destiny in the IPOP groups, as well as high satisfaction rates among those working in the IPOP environment. Higher salaries were also reported.

\section{Additional future steps}

An advantage of having both academically oriented child neurologists and child neurologists in IPOP environments is that it offers a variety of opportunities/models of practice for budding child neurologists. It encourages innovation and independent thinking with respect to the development of alternatives to the wRVU system of compensation, an unsustainable way to gauge compensation. These practice models may provide new and creative ways for alternative payment models, practice efficiencies, value-based medicine approaches, and performance or productivity models of compensation outside of the wRVU system.

The CNS should nurture, study, and document these different practice modes. CNS members could learn much about alternative models of care for our patients and how practices might be predicted to change with the ever-changing landscape of health care delivery. The CNS could become a vibrant partner with new members by providing resources, acting as a clearinghouse for innovative ideas, nurturing (perhaps with small grants) new ways to practice clinical medicine, and encouraging novel approaches to clinical research and the education of the future generation of physicians. Historically, academically based child neurologists have interacted and integrated well with IPOP pediatric neurologists, but there has been a trend away from such cooperative and mutually beneficial relationship. The CNS should work toward reestablishing such symbiotic ties.

We are in the best position to advocate for our profession. If we do not actively take part in the solution, we will have to accept what is handed to us. The new CPT codes (arriving January 1, 2020) could necessitate shorter appointments and there is an upcoming change in the reimbursement rate for current long-term video EEG monitoring (i.e., 95951). There are opportunities and much work to be done.

If wRVUs remain the sole basis of compensation, there will a continued high burnout rate and further loss of child neurologists, due to early retirement or a change in careers. The high workload, coupled with the possibility of declining compensation, does not bode well for the recruitment of future child neurologists. In addition, the reduction of child neurology training in pediatric residency programs has resulted in insufficient knowledge among pediatricians for managing even simple pediatric neurologic disorders. This is already creating high referral rates and insufficient access to child neurologists. These factors will only further exacerbate the critical workforce shortage of child neurologists.

If we act now, we can reverse this trend, specifically by partnering with the AAN and the American Academy of Pediatrics. 
With their robust resources, the CNS will be able to change the methodology by which compensation is determined and implement practical changes.

\section{Author contributions}

M.L. Zupanc: drafting/revising the manuscript, data acquisition, study concept or design, analysis or interpretation of data, accepts responsibility for conduct of research and final approval, acquisition of data, statistical analysis. B.H. Cohen: drafting/revising the manuscript, data acquisition, study concept or design, analysis or interpretation of data, accepts responsibility for conduct of research and final approval. P.B. Kang: drafting/revising the manuscript, data acquisition, study concept or design, analysis or interpretation of data, accepts responsibility for conduct of research and final approval. D.E. Mandelbaum: drafting/revising the manuscript, data acquisition, study concept or design, analysis or interpretation of data, accepts responsibility for conduct of research and final approval, acquisition of data, writing of paper. J. Mink: drafting/revising the manuscript, analysis or interpretation of data, accepts responsibility for conduct of research and final approval. M. Mintz: drafting/revising the manuscript, data acquisition, study concept or design, analysis or interpretation of data, accepts responsibility for conduct of research and final approval. A. Tilton: drafting/revising the manuscript, data acquisition, study concept or design, analysis or interpretation of data, accepts responsibility for conduct of research and final approval. W. Trescher: drafting/revising the manuscript, data acquisition, analysis or interpretation of data, accepts responsibility for conduct of research and final approval, acquisition of data, study supervision.

\section{Study funding}

No targeted funding reported.

\section{Disclosure}

M.L. Zupanc has research support for an investigatorinitiated retrospective study on infantile spasms from Mallinckrodt and clinical research support for investigational drug study from Zogenix, both paid to her employer; is on the Speaker's Bureau for Mallinckrodt, Eisai Inc., Aquestive Therapeutics, and Greenwich Pharmaceuticals, and has received honoraria; and is on the editorial board for Pediatric Neurology. B.H. Cohen receives research support from Stealth Biotherapeutics, BioElectron Technologies, Reath Pharmaceuticals, and Horizon Pharmaceuticals, all paid to his employer; is a consultant for Stealth Biotherapeutics and Modis Pharmaceuticals; is an educational speaker and CPT Advisor for the AAN; and has received honoraria for his services. P.B. Kang is a consultant for AveXis for a clinical trial and a consultant for ChromaDex; is on the advisory board for Sarepta Therapeutics; and is a coeditor for Muscle and Nerve, coeditor for Pediatric EMG (textbook), and author for UpToDate. D.E. Mandelbaum is on the Scientific Advisory Board of Teva Pharmaceuticals; is on the editorial boards of Pediatric Neurology and Journal of Child Neurology; occasionally performs medical expert consultations; and performs locum tenens (CompHealth). J. Mink has received research support from Abeona Inc. and Beyond Batten Disease Foundation; has research funding from the $\mathrm{NIH}$ and has received institutional support from the University of Rochester for research; serves as Associate Editor of Neurology and Associate Editor of Continuum; receives royalties from Elsevier, Inc.; and serves as a consultant for Abide Inc., Censa Inc., TEVA Inc., Bluebird Bio, and Neurogene. M. Mintz has functioned as principal investigator for clinical trials research contracted through the Clinical Research Center of New Jersey (CRCN) sponsored by the following companies: Aquestive Therapeutics, Curemark, Eisai Inc., Impax Laboratories, Neurim Pharmaceuticals, Nuvelution Pharma Inc., PhenoSolve, Sunovion, and Teva; is a consultant to Philips-Electrical Geodesics contracted through the Center for Neurologic and Neurodevelopmental Health $(\mathrm{CNNH})$ and is on the scientific advisory board of NeuroNeeds; serves on the editorial boards of the Journal of Child Neurology and Vision Development and Rehabilitation; has functioned as an expert witness in various litigation and mitigation cases, contracted through CNNH NeuroHealth; and is the Chief Medical Officer, Owner, and Founder of CNNH and CRCN, a board member of CNNH Management, Inc., and is President and Founder of NeurAbilities, a 501(3)c public charity. A. Tilton has worked with Ipsen Pharmaceutical on the development of a clinical study protocol and is a study investigator, with research support; is a consultant and educator for Eisai, Inc. and has received honoraria; and is a speaker on transitions of care for the Child Neurology Foundation. W. Trescher is an editor for the Journal of Pediatric Neurology. Go to Neurology.org/N for full disclosures.

\section{Publication history}

Received by Neurology March 20, 2019. Accepted in final form October 29, 2019.

\section{References}

1. Talbot SG, Dean W. STAT: Physicians Aren't “Burning Out”: They're Suffering from Moral Injury. 2018. Available at: www.statnews.com/2018/07/26/physicians-notburning-out. Accessed August 2019.

2. American Academy of Neurology Compensation and Productivity Report: 2016 Report on 2015 Data. Minneapolis: American Academy of Neurology; 2016.

3. Sullivan Cotter 2018 Survey: Pediatric Neurology. Available at: https://sullivancotter. com. Accessed August 2019.

4. ECG 2018 National Pediatric Subspecialty Survey: Pediatric Neurology. Available at: www.ecg.com. Accessed August 2019.

5. Kang PB, Bale JF, Mintz M, et al. The child neurology clinical workforce in 2015: report of the AAP/CHS Joint Taskforce. Neurology 2016;87:1284-1392.

6. Busis NA, Tait DS, Levin KH, et al. Burnout, career satisfaction, and well-being among US neurologists in 2016. Neurology 2017;88:1-12.

7. Zupanc ML, Tran LH, Mower A, et al. Cost effectiveness of multidisciplinary team approach in intractable epilepsy patients [abstract]. Presented at the 2015 meeting of the American Epilepsy Society; Philadelphia; 2015.

8. Wagner JL, Levisohn PM, Onufer CN, et al. Project access: strategies to improve care for children and youth with epilepsy: illustrations of recommendations in the IOM report on epilepsies. Epilepsy Behav 2013;29:121-127. 


\section{Neurology}

\section{Child neurology in the 21st century: More than the sum of our RVUs \\ Mary L. Zupanc, Bruce H. Cohen, Peter B. Kang, et al.}

Neurology 2020;94;75-82 Published Online before print December 24, 2019

DOI 10.1212/WNL.0000000000008784

This information is current as of December 24, 2019

Updated Information \&

Services

References

Citations

Subspecialty Collections

Permissions \& Licensing

Reprints including high resolution figures, can be found at:

http://n.neurology.org/content/94/2/75.full

This article cites 3 articles, 1 of which you can access for free at: http://n.neurology.org/content/94/2/75.full\#ref-list-1

This article has been cited by 7 HighWire-hosted articles: http://n.neurology.org/content/94/2/75.full\#\#otherarticles

This article, along with others on similar topics, appears in the following collection(s):

All Clinical Neurology

http://n.neurology.org/cgi/collection/all_clinical_neurology

All Practice Management

http://n.neurology.org/cgi/collection/all_practice_management

Billing

http://n.neurology.org/cgi/collection/billing

Health care reform

http://n.neurology.org/cgi/collection/health_care_reform

Models of care

http://n.neurology.org/cgi/collection/models_of_care

Information about reproducing this article in parts (figures,tables) or in its entirety can be found online at:

http://www.neurology.org/about/about_the_journal\#permissions

Information about ordering reprints can be found online:

http://n.neurology.org/subscribers/advertise

Neurology ${ }^{\circledR}$ is the official journal of the American Academy of Neurology. Published continuously since 1951, it is now a weekly with 48 issues per year. Copyright @ 2019 American Academy of Neurology. All rights reserved. Print ISSN: 0028-3878. Online ISSN: 1526-632X.

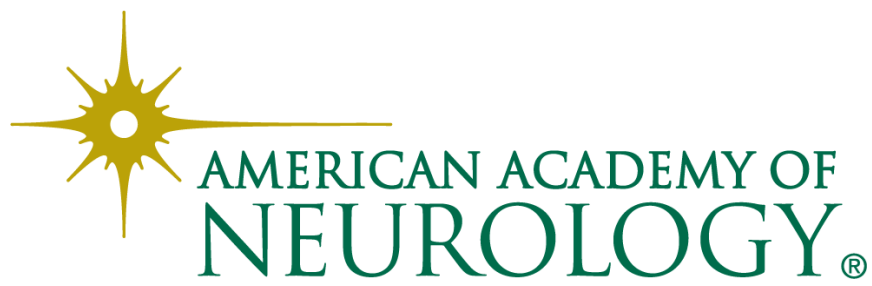

